\title{
Religion und Rechtsextremismus
}

\author{
Alexander Yendell · Kornelia Sammet
}

Angenommen: 10. November 2021 / Online publiziert: 16. Dezember 2021

(C) Der/die Autor(en) 2021

Mit den beachtlichen Erfolgen rechtsextremer Parteien und Bewegungen - nicht nur in Deutschland, sondern weltweit - rückt zunehmend das Thema Religion als ein Teilaspekt des Rechtsextremismus in den Vordergrund. Religion ist ein Element bzw. Bezugspunkt der Identitätspolitik ,rechtspopulistischer“ Parteien, in der ein Bedrohungsszenario durch andere Religionen - insbesondere durch den Islam - entworfen wird (Hidalgo et al. 2019). Dabei geht es vor allem um eine symbolische Bedrohung: Die Rede ist von ,westlichen“ und „christlichen“ Werten, die durch eine „Islamisierung“ in Gefahr gerieten. Allerdings finden sich nicht nur anti-islamische Positionierungen bei den extremen Rechten, sondern sowohl anti- als auch pro-christliche, -islamische und -jüdische Haltungen und Positionierungen (PfahlTraughber 2019; Salzborn 2018)

Gleichzeitig besteht zwischen Rechtsextremen und Kirchen seit jeher ein Spannungsfeld, sodass die Identifikation mit dem Christentum (dem ,,christlichen Abendland") eher diffus und ihre Authentizität bisweilen fraglich bleibt. Das zeigt sich beispielhaft am Umgang mit der Flüchtlingskrise. Während rechtsextreme Akteure darauf mit Rufen nach Abschottung und Ausgrenzung reagierten, wurde insbesondere in den Kirchen die ,Willkommenskultur“" praktiziert und auf die christlichen Werte Nächstenliebe, Gastfreundschaft und Barmherzigkeit hingewiesen.

Allerdings ist Religion im Zusammenhang mit Rechtsextremismus nicht erst seit Neuestem Gegenstand der akademischen und öffentlichen Diskussion. Bislang wurde in der Rechtsextremismusforschung vornehmlich auf Bezüge zu neopaganer Re-

\footnotetext{
Alexander Yendell $(\bowtie)$

Universität Leipzig, Leipzig, Deutschland

E-Mail: alexander.yendell@uni-leipzig.de

Kornelia Sammet

Deutsches Jugendinstitut e.V., Halle (Saale), Deutschland

E-Mail: sammet@dji.de
} 
ligiosität, Okkultismus und germanischer Mythologie abgehoben (z. B. Reich 1933; Goddrick-Clarke 2000; Wiedemann 2017). In der Forschung zum Nationalsozialismus wurde dieser sogar als eine Art (anti-christliche) Ersatzreligion diskutiert (Thomik 2009).

Darüber hinaus spielt Religion innerhalb der Autoritarismusforschung eine Rolle: Adorno et al. (1950) unterschieden typologisch zwischen dem „radikalen Christen“ einerseits, der eher intrinsisch religiös motiviert sei und zu Weltoffenheit und Toleranz neige, und der autoritären Persönlichkeit andererseits, die vor dem Hintergrund einer „Ich-Schwäche“ einen Hang zum Aberglauben habe, der eher exkludierend wirke (vgl. dazu auch Huber und Yendell 2019). In der für die Erforschung von Rechtsextremismus bedeutenden Vorurteilsforschung wird auf diese Unterscheidung zwischen ,intrinsischer“ und ,extrinsischer“" Religiosität (Allport/Ross 1967) rekurriert, obwohl ihre Erklärungskraft für die Analyse von Vorurteilen und Ungleichwertigkeitsvorstellungen bislang noch kontrovers diskutiert wird.

Vor diesem Hintergrund haben wir im Rahmen des 40. Kongresses der Deutschen Gesellschaft für Soziologie, der 2020 zum Thema „Gesellschaft unter Spannung“ stattfand, eine Veranstaltung der Sektion Religionssoziologie zu „Religion und Rechtsextremismus“ durchgeführt und uns danach entschlossen, die ausgearbeitete Fassungen der Vorträge zu veröffentlichen. Die Beiträge geben Antworten auf einige Forschungsfragen:

- Welche Bezüge bestehen zwischen Religion und Rechtsextremismus, und welche Theorien können diese Bezüge erklären?

- Welche Rolle spielt Religion für die Identitätspolitik von rechtsextremen Akteuren und Gruppen? Wie verbinden sich religiöse mit anderen (z.B. politischen) Elementen von rechtsextremen Identitätspolitiken und Programmatiken?

- Inwieweit bzw. in welcher Weise greifen rechtsextreme Akteure und Gruppen auf religiöse Lehren und Semantiken zur Legitimation zurück?

- Welche Formen von Religiosität und welche Glaubensüberzeugungen haben einen Einfluss auf rechtsextreme Einstellungen und Verhaltensweisen?

Insgesamt sieben Beiträge sind daraus entstanden, die diese Forschungsfragen diskutieren. Die Beiträge geben sehr vielfältige Einblicke in die Beziehungen von Religion und Rechtsextremismus und nutzen dabei unterschiedliche Forschungsmethoden und Datenmaterial.

Aletta Diefenbach untersucht, inwieweit Religion nicht nur eine säkulare und kulturelle Identität für extreme Rechte konstituiert, sondern auch tatsächlich ein glaubendes ,Wir“ bestärkt, das nicht nur anhand von öffentlichen Programmatiken und Diskursen, sondern auf der lebensweltlichen Ebene rechter Gruppierungen zu beobachten ist. Sie analysiert drei lokale Gruppen (AfD, Pegida, Identitäre Bewegung) und diskutiert deren Positionierungen zum Christentum.

Felix Schilk betrachtet in seinem Beitrag Form und Funktion religiös konnotierter Deutungsmuster in rechten Krisennarrativen. Dabei analysiert er in einer wissenssoziologischen Perspektive strukturelle Gemeinsamkeiten von Konservativismus, Rechtspopulismus und der Neuen Rechten sowie Ähnlichkeiten von rechten Krisennarrativen und apokalyptischen Texten. 
Christoph Bitzl und Michael Kurze befassen sich mit der Konstruktion von Freund- und Feindbildern bei der Alternative für Deutschland (AfD). Anhand einer Analyse von Programmen, Äußerungen und anderen Veröffentlichung der AfD diskutieren die Autoren, inwieweit sich die Partei einer bestehenden Feindlichkeit in der Bevölkerung gegen Muslime bedient und Religion als Vote-Seeking-Strategie instrumentalisiert.

Niklas Herrberg nimmt den Zusammenhang zwischen der Reichbürgerszene, religiösen Semantiken und Verschwörungssemantiken in den Blick und untersucht, welche Rolle Religion für deren Argumentation spielt. Er interessiert sich zum einen für die Funktion der Religion für die Reichsbürger und zum anderen für den Eingang religiöser Semantiken in ihre Argumentation.

Carsten Heinze und Marcus Stiglegger gehen der Frage nach, inwiefern religiöse Haltungen, spirituelle Bezüge sowie Rechtsextremismus in verschiedenen historischen Jugend-/Subkulturen zu finden sind und auf welche Weise sie miteinander verknüpft sind. Die Autoren beschäftigen sich dabei mit den Szenen des GothicRock, des Neofolk und des Black Metal.

Verena Schneider, Gert Pickel und Cemal Öztürk beschäftigen sich mit dem Zusammenhang von Religion, Vorurteilen und Rechtsextremismus. Auf Grundlage der statistischen Analyse von repräsentativen Umfragedaten stellen sie die ambivalente Rolle von Religion und verschiedenen Formen von Religiosität heraus, die einerseits inkludierend sein können und mit Toleranz einhergehen und andererseits exkludierend wirken sowie Vorurteile und Rechtsextremismus beflügeln können.

Auch der letzte Beitrag von Nils Friedrichs setzt sich auf Grundlage statistischer Datenanalysen mit dem Zusammenhang von Religiosität und Rechtsextremismus auseinander. Dabei thematisiert der Autor verschiedene Aspekte des Glaubens, wie der an ein Leben nach dem Tod und die Hölle, sowie des Atheismus im Zusammenhang mit Rechtsextremismus.

Insgesamt lässt sich nach Lektüre der Beiträge konstatieren, dass Religion bedeutende und vielfältige Funktionen im Zusammenhang mit Rechtsextremismus einnehmen kann. Sie dient rechten Akteuren zur Instrumentalisierung zum Zwecke der identitätsstiftenden Abgrenzung von Feinden. Das alleine wäre aber zu kurz gegriffen, da religiöse Bezüge und Semantiken im Kontext des Rechtsextremismus auch in den Krisennarrativen der (Neuen) Rechten eine Rolle spielen und verschiedene Formen von Religiosität sowie Aspekte des Glaubens rechtsextreme Einstellungen befördern oder aber auch verhindern können. Die Beiträge zeigen, wie komplex der Aspekt der Religion im Rechtsextremismus ist und dass dieser sowohl in der Forschung als auch in der Prävention von Rechtsextremismus nicht weiter vernachlässigt werden sollte.

Danksagung Wir danken allen Autorinnen und Autoren für die anregenden Diskussionen während der Sektionsveranstaltung sowie für ihre Anstrengungen, die Beiträge rechtzeitig für die Publikation fertigzustellen. Ebenso danken wir der Redaktion und den Herausgeber:innen der ZRGP für die reibungslose Organisation und natürlich den anonymen Gutachterinnen und Gutachtern für ihre hilfreichen Kommentare und Verbesserungsvorschläge.

Funding Open Access funding enabled and organized by Projekt DEAL. 
Open Access Dieser Artikel wird unter der Creative Commons Namensnennung 4.0 International Lizenz veröffentlicht, welche die Nutzung, Vervielfältigung, Bearbeitung, Verbreitung und Wiedergabe in jeglichem Medium und Format erlaubt, sofern Sie den/die ursprünglichen Autor(en) und die Quelle ordnungsgemäß nennen, einen Link zur Creative Commons Lizenz beifügen und angeben, ob Änderungen vorgenommen wurden.

Die in diesem Artikel enthaltenen Bilder und sonstiges Drittmaterial unterliegen ebenfalls der genannten Creative Commons Lizenz, sofern sich aus der Abbildungslegende nichts anderes ergibt. Sofern das betreffende Material nicht unter der genannten Creative Commons Lizenz steht und die betreffende Handlung nicht nach gesetzlichen Vorschriften erlaubt ist, ist für die oben aufgeführten Weiterverwendungen des Materials die Einwilligung des jeweiligen Rechteinhabers einzuholen.

Weitere Details zur Lizenz entnehmen Sie bitte der Lizenzinformation auf http://creativecommons.org/ licenses/by/4.0/deed.de.

\section{Literatur}

Adorno, T.W., et al. 1950. The authoritarian personality. : Harper und Brothers.

Allport, G. and Ross, J.M. (1967) Personal Religious Orientation and Prejudice. Journal of Personality and Social Psychology 5(4):432-443. https://doi.org/10.1037/h0021212

Goodrick-Clarke, N. 2000. Die okkulten Wurzeln des Nationalsozialismus. Graz: Stocker.

Hidalgo, O., Hildmann, P. W. \& Yendell, A. (Hg.) (2019). Religion und Rechtspopulismus. Argumentation kompakt 3, 1-6.

Huber, S., und A. Yendell. 2019. Does Religiosity matter? Explaining right-wing extremist attitudes and the vote for the Alternative for Germany (AfD). Religion and Society in Central and Eastern Europe 15(2):63-85. https://doi.org/10.20413/rascee.2019.12.1.63-82.

Pfahl-Traughber, A. 2019. Der Extremismus der Neuen Rechten. Eine Analyse zu Diskursthemen und Positionen. Berlin Heidelberg: Springer.

Reich, W. 1933. Die Massenpsychologie des Faschismus. Kopenhagen: Sexpol.

Salzborn, S. 2018. Rechtsextremismus. Erscheinungsformen und Erklärungsansätze. : BpB.

Thomik, J. (2009). Nationalsozialismus als Ersatzreligion: Die Zeitschriften „Weltliteratur“ und „Die Weltliteratur“ (1935/1944) als Träger nationalsozialistischer Ideologie; zugleich ein Beitrag zur Affäre Schneider/Schwerte. Einhard.

Wiedemann, P. 2017. Das Verhältnis der extremen Rechten zu Religion. In Handbuch Rechtsextremismus, Hrsg. F. Virchow, M. Langebach, und A. Häusler, 511-532. Berlin Heidelberg: Springer VS. 